ENSAYOS

\title{
LAS CONCEPCIONES QUE ORIENTAN LAS PRACTICAS EVALUATIVAS DE LOS PROFESORES: UN PROBLEMA A DEVELAR*
}

\author{
Teachers' conceptions that inform their assessment practices:
}

a problem to be disclosed

\author{
Marcia Prieto $^{1}$, Gloria Contreras ${ }^{2}$ \\ ${ }^{1}$ Universidad de Valparaíso, 1 Norte 2431, Viña del Mar, Chile. marciaprietop@gmail.com \\ ${ }^{2}$ Pontificia Universidad Católica de Valparaíso, Avenida Brasil 2950, Valparaíso, Chile. gcontrer@ucv.cl
}

\section{Resumen}

La evaluación escolar se ha convertido en el último tiempo en un tema de gran preocupación, debido a la creciente evidencia que señala que se desarrolla con sentidos y prácticas que, más que apoyar al aprendizaje de los estudiantes, lo obstaculiza. En este artículo se desarrolla una revisión analítica de los sentidos asignados a la evaluación en la actualidad. Se destacan resultados de estudios nacionales e internacionales referidos a los sentidos y concepciones que estarían orientando y determinando las prácticas evaluativas de los profesores básicos de matemáticas y lenguaje, dada su importancia y efectos posteriores en los estudiantes. Se concluye la necesidad de develar e investigar las concepciones subyacentes, revelando los sentidos asignados a sus prácticas evaluativas, dadas las importantes repercusiones en el itinerario de sus estudiantes.

Palabras clave: evaluación del aprendizaje, concepciones, creencias, conocimiento profesional docente, prácticas evaluativas.

\begin{abstract}
School assessment has turned into an important issue to be analysed. A growing amount of evidence has diclosed the fact that its direction and practices are not favouring students learning but rather obstructing it. This paper develops an analitical review of findings reported in some national and international studies dealing with teachers beliefs about their meanings of assessment. Special attention has been given to Mathematics and Literacy primary teachers beliefs, mostly because of the subsecuent long lasting effects in students. On the bases of the reported evidences it is concluded the necessity of researching and disclosing teachers ideas about assessment, so as to bring out the impact of their beliefs in their their practices, taking into account the critical repercussions in the students school itinerary.

Key words: school assessment, teachers beliefs, teachers professional knowledge; assessment practices.
\end{abstract}

\footnotetext{
* $\quad$ Proyecto FONDECYT 1070324.
} 


\section{INTRODUCCION}

Tradicionalmente se ha considerado la evaluación como un proceso técnico de verificación de resultados de aprendizaje acorde con objetivos predeterminados. Sin embargo, la evaluación escolar es un proceso mucho más complejo, pues en él confluyen una gran variedad de aspectos, algunos de ellos bastante explícitos y otros menos advertidos, dado que están implícitos. Los primeros provienen desde la escuela y están asociados a los contenidos educativos, normas, regulaciones, criterios, procedimientos, exigencias administrativas y condiciones de trabajo en las aulas, aspectos que no sólo orientan la evaluación sino que también la afectan. Los implícitos provienen desde los propios profesores quienes aplican criterios e implementan prácticas docentes relacionadas con sus concepciones respecto de enseñanza y evaluación, valorando de una u otra manera sus fines, sentido y trascendencia y determinando, en gran medida, la naturaleza de sus prácticas de enseñanza y evaluativas e incidiendo de manera crítica en los resultados de los procesos formativos.

Dado lo anterior, no es posible considerar la enseñanza y la evaluación como procesos neutros o inocuos, no sólo porque influyen en la calidad de los procesos formativos y producen efectos en los estudiantes que les impactan de manera crítica sino que, dado que es una actividad esencialmente subjetiva y valorativa, constituyen un reflejo de las concepciones de los profesores, entre las que se encuentran las creencias y/o conocimientos especializados. Estas concepciones representan una base relevante para la práctica profesional de los profesores, dado que operan como el fundamento central que guía sus prácticas las que, no obstante su importancia, han sido relativamente poco reconocidas y mucho menos investigadas. Este hecho ha producido problemas y controversias originadas por la manera como los profesores están entendiendo la evaluación, dado que les conduce a seleccionar determinadas prácticas e instrumentos de gran impacto en la calidad de los procesos formativos con efectos no siempre deseados en los estudiantes. Dado lo anterior se deduce la importancia de conocer las concepciones, analizar el sentido que le asignan los profesores y develar, así, las racionalidades que las informan (Borko 1997).

Evaluación escolar: sentido y racionalidades que la orientan. La evaluación es un término polisémico que tiene su origen en procesos sociales e históricos particulares y que no tiene identidad disciplinaria, dado que se emplea en diversos campos, uno de los cuales es el educativo. La evaluación en este campo forma parte de la Didáctica, disciplina que estudia la enseñanza como fenómeno, desplegando su complejidad y relevando los múltiples y diversos aspectos que la informan, cruzan y conforman. De acuerdo a los planteamientos actuales, la evaluación es un proceso complejo orientado a recoger evidencias respecto del aprendizaje de los estudiantes de manera sistemática para emitir juicios en pos de un mejoramiento tanto de la calidad del aprendizaje como de la enseñanza. Este proceso se apoya en dos elementos fundamentales: los criterios de evaluación y la recolección de información. Respecto de este último, la información puede ser recogida tanto a través de instrumentos formales (pruebas escritas, trabajos, disertaciones, etc.) como informales, dado que el profesor puede obtener evidencias respecto de los aprendizajes de los estudiantes a través de la simple observación de las interacciones acaecidas cotidianamente en las aulas. 
Aun cuando la evaluación representa un proceso sustantivo del proceso educativo, no ha alcanzado el mismo rango de centralidad que han tenido, por ejemplo, los aspectos curriculares. Si bien en la actualidad es posible observar un cambio en este sentido, ello ha acontecido como resultado de una necesidad ajena a la preocupación por mejorar los aprendizajes y la formación de los estudiantes. De hecho, su relevancia obedece más bien a aspectos relacionados con "la certificación y legitimación de conocimientos y la convalidación de un mínimo de aprendizajes curricularmente previstos" (Palau de Mate 2005: 98). Esta situación ha tenido como resultado que la evaluación ha transformado el debate educativo desde ser un problema conceptual hacia convertirlo en un problema técnico y de control (Díaz Barriga 1993).

En efecto, la mayoría de las prácticas docentes se ha estructurado en función de la evaluación, pero privilegiando la reproducción y control del conocimiento de los estudiantes, en desmedro de su producción o construcción y/o del desarrollo de sus habilidades cognitivas superiores. Así lo confirman los resultados de algunas investigaciones que concluyen que las concepciones que orientan las prácticas evaluativas de los profesores corresponden a enfoques mayoritariamente instrumentales y memorísticos que priorizan los resultados alcanzados en términos del rendimiento, la capacidad reproductiva y esfuerzo individuad (Stiggins 2004; Celman 2005). Esto ha significado una reducción del proceso evaluativo al otorgarle el sentido de simple comparación de respuestas según correspondan o no a lo transmitido por el profesor o el texto (Litwin 2005). Sin embargo, el sentido de la evaluación es bastante más comprehensivo que esto, por lo que es necesario analizarlo.

Sentido de la evaluación. El sentido de la evaluación desde una perspectiva didáctica se asocia, según algunos autores, a la emisión de juicios valorativos sobre el trabajo y acciones de los estudiantes, construidos desde los marcos axiológicos y epistemológicos del profesor y en el que confluyen, por tanto, aspectos subjetivos. Para otros, incluye también revisar y plantear interrogantes a los profesores acerca de sus prácticas (Celman 2005; Litwin 2005; Camilloni 2005; Darling-Hammond 2001; Eisner 1994). No obstante lo anterior, la evaluación sigue siendo considerada, mayoritariamente, como un proceso desvinculado de la enseñanza y destinado simplemente a medir, acreditar o certificar los resultados de aprendizajes y, por lo tanto, como un "acto final desprendido de las acciones propias de la enseñanza y el aprendizaje" (Celman 2005: 37). Desde esta mirada, la enseñanza se reduciría a un proceso lineal de entrega de insumos y obtención de productos y el aprendizaje estaría referido a un simple proceso de acopio de datos e informaciones, para ser reproducidas lo más fielmente posible, asumiendo que todo lo que se enseña se aprende (Santos Guerra 1996). El sentido de la evaluación, de este modo, estaría centrado en la medición del logro de objetivos curriculares, a partir de instrumentos que permitieran verificar de manera empírica los objetivos alcanzados por el estudiante (Bliem y Davinroy 1997).

Este sentido instrumental y supuestamente objetivo representa una visión restringida del proceso evaluativo, pues revelaría aspectos parciales del nivel de aprendizaje del estudiante, esto es, sólo permitiría conocer qué sabe, pero no proporcionaría información respecto de qué forma lo sabe, por qué lo sabe, qué no sabe o por qué no lo sabe. La evaluación, desde este enfoque, se convierte en un fin en sí misma, en una medida de control y en un instrumento punitivo, que ignora las peculiaridades de cada estudiante 
y que tiene como propósito comprobar el aprendizaje para otorgar una calificación que sólo indica cuánto sabe el estudiante. Es decir, la evaluación se remitiría sólo a 'contar' los aprendizajes (Stiggins 2006)

Por el contrario, la mirada más inclusiva establece que la evaluación, como ya se ha señalado, no sólo proporciona información respecto del nivel de aprendizaje de los estudiantes, sino que suministra, también, indicios empíricos acerca de la eficacia de la enseñanza (Jackson 2002). Por lo tanto, el proceso evaluativo y, de manera más específica sus resultados, permitirían al profesor no sólo visualizar la evolución de cada niño con respecto a su aprendizaje, identificar sus necesidades y detectar sus dificultades sino que, a su vez, le permitiría establecer relaciones entre estas dificultades y las concepciones y prácticas docentes a partir de las cuales se enseñaron. Es decir, la evaluación permitiría no sólo 'contar' los aprendizajes sino que también promoverlos, recuperando su más pleno sentido formativo, dado que los profesores no sólo podrían analizar las dificultades propias del contenido disciplinario y/o la pertinencia de los medios e instrumentos seleccionados, sino que también les facilitaría la identificación de los supuestos, creencias y conocimientos que los están informando.

Este ejercicio requiere que los profesores desplieguen sus capacidades metacognitivas y desarrollen un proceso reflexivo de autoevaluación sostenido en el tiempo, por medio del cual pueden obtener información relevante sobre estos aspectos. Ello, porque toda práctica en cualquier contexto es una experiencia provisoria y, como tal, debería estar sujeta a análisis y revisión sistemática a partir de detectar y reflexionar críticamente acerca de su sentido y sus efectos. Asimismo, porque favorece el desarrollo de procesos formativos a partir de la reflexión constante, lo que permite tanto la inmersión de los profesores en su mundo de concepciones como la visibilización de sus propias prácticas, apoyando su transformación de manera razonada, pertinente y viable (Stenhouse 1998). En definitiva, este proceso reflexivo les ayuda a reorientar y determinar el curso de la enseñanza y de la evaluación, implementando las acciones pedagógicas suplementarias tendientes a apoyar a los estudiantes en la superación de los problemas y dificultades detectadas, más que a penalizarlos por sus debilidades (Martínez 2004; Durán 2001; Montero 2001; Shön 1992).

Si se explicitaran las concepciones que informan las prácticas evaluativas, los profesores podrían descubrir la heterogeneidad de concepciones que están orientando sus prácticas e instrumentos evaluativos, los que suelen ser muy diversos $\mathrm{y}$, en ocasiones, opuestos. Al respecto se puede mencionar, a modo de ejemplo, que muchos profesores creen que la aplicación de pruebas estandarizadas constituye un buen procedimiento para conocer lo que los estudiantes saben. Otros, sin embargo, sostienen que estas pruebas anulan las subjetividades de los estudiantes e impiden valorar sus aportes para una mejor comprensión de los complejos problemas asociados a las tareas de enseñar y aprender (Eisner 1998). Asimismo, algunos profesores creen que la evaluación debe privilegiar la creatividad o la capacidad analítica de los estudiantes; otros creen que es importante identificar la exactitud de las respuestas o el progreso alcanzado acorde con los objetivos prescritos (Torrance y Pryor 1998). Estas creencias revelan concepciones sobre enseñanza y evaluación totalmente antitéticas que producen efectos también opuestos en los estudiantes, que los alienan o los emancipan.

De lo anterior se podría deducir que los profesores someten a sus estudiantes a procesos evaluativos cuyo sentido estaría informado primordialmente por sus concepciones 
y preferencias, sin atender a la naturaleza del contenido a evaluar o las características, dificultades y posibilidades de los estudiantes y del contexto en el cual se desarrollan. Esta situación acarrea efectos importantes en los estudiantes, algunos de los cuales pueden traer consecuencias bastante críticas y desfavorables para ellos. Una de las más sustantivas está referida a la imagen de sí mismo que se construyen, la que puede ser distorsionada y/o generar sentimientos de sobrevaloración, dependencia o inseguridad, obstaculizando, así, la construcción de su identidad personal. Al respecto, es necesario precisar que la identidad y autoestima de los estudiantes se ven afectadas en gran medida por sus éxitos y fracasos escolares (objetivados en los resultados de las evaluaciones), pues estos se constituyen como "los nutrientes principales en el desarrollo de la personalidad" (Litwin 2005: 21).

Se podría deducir que la evaluación constituye para los estudiantes, no sólo una actividad administrativa que acredita el nivel de sus conocimientos, sino que representa una experiencia personal y emocional de efectos substanciales y de largo alcance, dado que condicionarán el desarrollo de sus habilidades necesarias para progresar debidamente en su itinerario escolar, determinarán su futuro escolar e incidirán fuertemente en la construcción de sus identidades (Litwin 2005; Earl y LeMahieu 2003). Ello, porque los procesos escolares constituyen los caminos por los cuales transitan los estudiantes en pos del encuentro con experiencias que los interpelen e interroguen y que, en el proceso de su develamiento, construyan sus subjetividades (Frigeiro 1998).

Asimismo, la creencia de otros profesores referida a que los estudiantes provenientes de los sectores de mayor vulnerabilidad carecerían de bienes materiales y simbólicos, les induce a bajar sus expectativas respecto de sus posibilidades de éxito, lo que incide poderosamente en su rendimiento (Duschatsky 1999). Ello podría conducir a que muchos estudiantes con bajas calificaciones llegaran a pensar que el éxito en la escuela es un privilegio de algunos, de aquellos supuestamente mejor dotados y/o con menos limitaciones, aun cuando podría ser el resultado de procesos evaluativos mal diseñados o indebidamente desarrollados. Asimismo, podrían sentirse excluidos o marginados anticipadamente de la escuela, lo que podría conducirlos, finalmente, al fracaso escolar y eventualmente a la deserción, con todas las secuelas negativas que esto implica para su futuro educacional y laboral. Los resultados alcanzados por los estudiantes, en consecuencia, no sólo constituirían un reflejo de sus niveles de comprensión o de los obstáculos epistemológicos que les impiden comprender determinados contenidos, sino que también revelarían las concepciones sustentadas por los profesores acerca del sentido y naturaleza de la evaluación y las racionalidades que las informan (Litwin 2005; Durán 2001; Gómez Chacón 2000; Peterfalvi 1997; Bachelard 1993).

Racionalidades que orientan la evaluación. Es posible identificar dos concepciones de enseñanza con finalidades acordes con la racionalidad que la informan: la técnico-instrumental y la formativa-emancipadora. Desde la racionalidad instrumental, la enseñanza constituye un camino para solucionar los problemas educativos mediante la aplicación de técnicas y procedimientos que se justifican por su capacidad para conseguir efectos y resultados deseados. Los estudiantes son considerados meros receptores pasivos de información, la que deben reproducir lo más fielmente posible, constituyéndose, por lo tanto, en simples objetos de acciones técnicas planificadas (Prieto 2005). Los profesores son los encargados de entregar y distribuir la información, de manera tal que les permita 
realizar un adecuado control de su "apropiación". La evaluación se conceptualiza como un proceso técnico de "certificación de los productos o resultados de aprendizajes planteados en programas y planes oficiales" (Palau de Mate 2005: 98). Es decir, se le asigna la función de control, selección, jerarquización y acreditación, a partir de la medición del nivel de aprendizaje de los estudiantes. El testimonio de un profesor reportado en un estudio acerca del tema lo confirma: "Yo establezco ciertos objetivos y después pongo una marca para saber cuánto han producido los niños al respecto" (Torrance y Pryor 1998: 22).

Desde la racionalidad formativa-emancipadora, la enseñanza se entiende como la implementación de una amplia y diversificada gama de experiencias formativas, sustentadas sobre el conocimiento personal de los estudiantes, modos de pensar y contextos que los enmarcan. De este modo, representa el camino que propicia el desarrollo de las habilidades cognitivas y sociales de los estudiantes que los volverán personas autónomas y conscientes de sus responsabilidades sociales (Freire 2002; Eisner 1994; Carr 1999). Los estudiantes son considerados sujetos centrales y protagonistas de sus propios procesos de desarrollo, a partir de su activa y sostenida participación. El profesor se convierte, por lo tanto, en un facilitador de las condiciones y oportunidades para que los estudiantes aprendan a pensar por sí mismos y se les faculte para tomar sus propias decisiones (Freire 2002; Darling-Hammond 2001; Shor 1992). La evaluación, denominada auténtica, enfatiza la función formativa del proceso educativo y se constituye como una práctica tendiente tanto a conocer el nivel de comprensión de los estudiantes y nivel de desarrollo de habilidades y destrezas propias de un determinado contenido enseñado como a reflexionar acerca de las prácticas implementadas por los profesores, detectando y explicitando los fundamentos epistemológicos que las orientan.

Resulta interesante mencionar un estudio que consigna expresiones de algunos profesores a partir de las cuales se puede inferir una comprensión de evaluación como proceso formativo $\mathrm{y}$, por lo tanto, como un medio que proporciona información para tomar decisiones encaminadas a mejorar los procesos de enseñanza y aprendizaje. Así lo revelan las palabras de un profesor en un estudio: "La evaluación no está ligada necesariamente a lo que el niño está haciendo, sino que debemos conocer cómo puede seguir adelante y cuáles son sus necesidades especiales" (Torrance y Pryor 1998: 25). Otro profesor sostenía en ese mismo estudio: "Yo trato de ser lo más positivo que puedo. Si no han comprendido, les digo que haremos un nuevo intento, que yo podría intervenir y ayudarlos verdaderamente a hacerlo de nuevo (ibídem 36). Otros profesores incorporan la perspectiva mutidimensional del proceso educativo, lo que les permite aprehender la riqueza y profundidad de los aprendizajes, conceptualizando 'evaluación' como un proceso dinámico e interactivo de construcción conjunta de conocimiento, tal como lo confirman las siguientes expresiones: "Es muy importante conocer lo que en realidad saben los niños, suponemos que son del tipo promedio, pero muchas veces ellos nos sorprenden y saben mucho más de lo que se le ha enseñado" y de esta manera "Ellos (los estudiantes) avanzan y retroceden, pero lo que importa es el conocimiento que uno construye con los niños” (ibídem 37).

Dada la existencia de estas racionalidades y sentidos diversos respecto del proceso evaluativo es posible entender que existan evaluaciones del aprendizaje y evaluaciones para el aprendizaje: las primeras controlan el nivel de aprendizaje de los estudiantes, las segundas los favorecen, dependiendo de las concepciones y prácticas de los profesores 
(Stiggins 2006). Mirado desde otra perspectiva, estas significaciones podrían establecer una distinción entre los profesores que creen que deben enseñar para evaluar y aquellos que creen que evalúan para enseñar mejor (Bolívar 2000). De lo anterior, se deduce la importancia de conocer las concepciones que están orientando las prácticas evaluativas de los profesores de manera de comprender su sentido y objetivos.

Concepciones de los profesores. Es necesario destacar la emergencia de un creciente interés por conocer el pensamiento de los profesores, dado que éste representa un conjunto de estructuras internas a partir de las cuales realizan sus prácticas sociales y de manera específica, los docentes. Un número importante de investigaciones ha intentado identificar este pensamiento y sus resultados han configurado una base de conocimiento que ha permitido conocer aspectos tradicionalmente ignorados y conocer sus efectos en los estudiantes (Bolívar y Segovia 2004; Durán 2001; Marcelo 2002; Torrence y Pryor 2001; Delandshere y Jones 1999; Eraut 1994). En efecto, estos estudios identifican una gran variedad de prácticas docentes, las que sólo pueden ser comprendidas en referencia tanto a las concepciones e intenciones que las orientan como al contexto particular en el que se despliegan, el que está enmarcado por dimensiones sociales, culturales históricas y políticas que las condicionan (Carr 1999).

Independientemente de cómo se denomine esta base de conocimientos y significaciones de los sujetos, se constituyen como organizadores implícitos referidos a creencias, significados, conceptos, proposiciones, imágenes mentales y preferencias que influyen tanto en la manera de percibir la realidad como en las prácticas que implementan (Moreno y Azcárate 2003). En el caso de los profesores, estas estructuras mentales o concepciones abarcan tanto sus conocimientos profesionales como sus creencias, las que se entrelazan en la experiencia profesional y se objetivan en el contacto con la realidad escolar. Así se constituyen como los referentes a los que acuden para organizar y tomar las consiguientes decisiones respecto de su trabajo profesional, las que demuestran tener gran incidencia en la calidad de los aprendizajes de los estudiantes (Marcelo 2002; Durán 2001; Ponte 1992).

Conocimiento profesional. Las prácticas evaluativas pueden estar orientadas por redes semánticas y/o conceptuales referidas a la naturaleza y sentido de la disciplina, su enseñanza en general y evaluación en particular, afectando y calificando ambas actividades. Esto es lo que se denomina conocimiento profesional docente y que constituye un conjunto de saberes pedagógicos, criterios profesionales y argumentaciones teóricas explícitas o implícitas de naturaleza diferente. Es decir, constituirían el marco de referencia organizativo de las prácticas docentes que influyen y afectan los procesos formativos en las aulas y a partir de las cuales los profesores los perciben, organizan y ejecutan.

Estas redes teóricas han sido construidas en su formación inicial y a partir de las distintas experiencias de su desarrollo profesional. Se ha demostrado, a modo de ejemplo, que la experiencia del profesor es un factor importante para efectos de la construcción de estos marcos de referencias, dado que se observan discrepancias entre las concepciones y prácticas de aula de los profesores novatos y las de los experimentados (Avalos 2003; Mellado 1996). Esto indica que la simple construcción de conocimiento profesional no se relaciona necesariamente con una capacidad para desarrollar procesos formativos en consonancia con su naturaleza y finalidades, pues esta transferencia requiere la construc- 
ción de esquemas mentales que orienten la acción práctica acorde con las concepciones $\mathrm{y}$ funciones asignadas a este proceso.

El conocimiento profesional ha sido definido y categorizado por diversos autores desde enfoques diversos, aun cuando se observa una cierta unanimidad entre ellos, dado que todos integran aspectos asociados al conocimiento pedagógico general, al disciplinario y al didáctico del contenido. El conocimiento pedagógico general está relacionado con la historia, filosofía, sociología y psicología de la educación; los principios generales de la enseñanza y del aprendizaje; el conocimiento del contexto escolar, la planificación, evaluación y prácticas pedagógicas en general. Por su parte, el conocimiento disciplinario se refiere al cuerpo de conocimientos especializados asociados a la disciplina a enseñar (objetivada en planes y programas de estudio), que requieren ser adaptados a las condiciones particulares del contexto cultural de la escuela y de los estudiantes (Marcelo 2002; Montero 2001; Chevallard 1999; Shulman 1987). Este conocimiento es fundamental, dado que "conocer algo nos permite enseñarlo y conocer un contenido con profundidad significa estar mentalmente organizado y bien preparado para enseñarlo de una forma general" (Buchmann 1984: 37, citado por Marcelo 2002).

Finalmente, el conocimiento didáctico del contenido representa la articulación entre el conocimiento disciplinario y conocimiento pedagógico. Es decir, define cómo enseñarlo y se constituye como el puente entre el significado del contenido disciplinario y la construcción realizada por los estudiantes de ese significado. En otras palabras, permite reordenar y simplificar los contenidos disciplinarios para transformarlos en conocimiento enseñable que propicie su comprensión por parte de los estudiantes. Se asume que los profesores que trabajan profesionalmente conocen, relacionan y aplican estos conocimientos en las prácticas docentes, integrándolos como un todo orgánico y entendiendo su dinámica como una unidad en acción (Martínez 2004; Shön 1992).

Creencias de los profesores. Existe gran dispersión semántica en la nominación del término 'creencias', dado que han sido nominadas, indistintamente, como actitudes, valores, juicios, axiomas, opiniones, ideologías, percepciones, preconcepciones, disposiciones, procesos mentales internos, reglas de la práctica, principios prácticos, etc. No obstante lo anterior, existe consenso respecto de entenderlas como construcciones mentales válidas para los actores, que informan y afectan de manera directa sus prácticas sociales.

Las creencias, en general representan construcciones realizadas por los sujetos que les facilitan la comprensión del mundo en el que viven, que inciden en sus comportamientos y median su comprensión (Martínez Padrós 2003). Las creencias no necesitan ser consensuadas para ser consideradas válidas, como tampoco requieren de reglas lógicas para determinar su correspondencia con situaciones reales. De este modo, se constituyen como verdades idiosincráticas que no requieren una condición de verdad contrastada, dado que representan datos, supuestos y opiniones propias o transmitidas por otros y surgidas desde los saberes del sentido común (Carr y Kemmis 1988).

En general, el sentido común representa el referente central del comportamiento de las personas en la vida cotidiana, pues les familiariza con los significados de los conceptos y categorías que les permiten comprender la realidad y dar significado a la experiencia. Sin embargo, las creencias pueden diferir sustancialmente de la realidad, pues se generan a partir de experiencias acumuladas episódicamente en la memoria, pues "el conocimiento de un tema se diferencia de los sentimientos que tengamos sobre ese tema" (Pajares 1992: 
309). Están estructuradas sobre la base de elementos afectivos y evaluativos, tipificando no sólo 'lo que es' sino que también 'lo que debería ser' (Jackson 2002; Nespor 1987). De esta manera, representan redes semánticas personales que configuran un repertorio de supuestos y respuestas rutinarias frente a los problemas profesionales, sin base teórica que las sustenten. En este sentido, son simples disposiciones para la acción, aun cuando se constituyen como determinantes críticos del comportamiento, expresados en tiempos y contextos específicos (Ponte 1999).

No obstante lo anterior, las creencias representan para muchos profesores una base relevante para desarrollar sus prácticas profesionales, las que se han arraigado de tal manera en su inconsciente que les dificulta reconocer la existencia de otras formas de realizarlas y, por lo tanto, acuden a ellas como respuestas válidas, especialmente cuando se ven enfrentados a situaciones desconocidas o confusas. Este hecho en muchas ocasiones les impide aplicar una estructura adecuada de saberes, dadas las limitaciones, problemas e inconsistencias propias de su naturaleza (Nespor 1987). Sin embargo, es justo reconocer el trabajo silencioso y sistemático de muchos profesores que luchan contra ellas y logran superarlos a partir de procesos conscientes y reflexivos (Kaplan 2004).

Ahora bien, es necesario precisar que los profesores no construyen sus creencias de manera intencionada y consciente, por el contrario, las han internalizado en sus estructuras cognitivas y emocionales de manera inconsciente, como resultado de una gran diversidad de experiencias articuladas: las personales y las escolares. Las personales les han permitido construir una determinada visión de sí mismos y del mundo, lo que ha resultado en la generación de ideas sobre la familia, la cultura y las relaciones entre escuela y sociedad, entre otros aspectos (Marcelo 2002; Durán 2001). A su vez, las experiencias escolares acaecidas en el contexto de la cultura escolar en la que se desempeñan les han facilitado la construcción social de creencias respecto de la educación, la enseñanza, el aprendizaje, la evaluación y la disciplina, entre otros aspectos (Wang 2004; Delanshere y Jones 1999).

Estas experiencias escolares, en ocasiones, han resultado en la construcción de expectativas y prejuicios difíciles de remover, por lo que se mantienen generalmente sin cambios y acompañan a los profesores de manera constante durante sus prácticas de enseñanza (Stiggins 2004; Durán 2001; Liston y Zeichner 1993). Así lo comprueban los resultados de un estudio en el que muchos profesores otorgaron bajas calificaciones a los escolares provenientes de los sectores populares etiquetando sus resultados como 'simplones', 'vulgares' o 'empalagosos', como producto de sus creencias y prejuicios respecto de estos estudiantes y no necesariamente de la validez de sus construcciones (Kaplan 2004, citando a Bourdieu y San Martín 1975).

Ahora bien, existen diversas creencias respecto del sentido y naturaleza de la evaluación, que les conducen a desarrollar prácticas acordes con éstas. En efecto, se ha detectado que los profesores que sostienen creencias tradicionales respecto de este proceso y la entienden como un instrumento de control y un medio de información con un alto grado de objetividad, enfatizan las respuestas correctas y la realización de tareas simples (Stiggins 2004). Otros, sin embargo, creen que constituye una oportunidad para realizar una mirada introspectiva sobre la propia docencia, lo que les faculta para orientar y determinar el curso de sus tareas docentes, por lo que relevan aspectos formativos del proceso evaluativo, enfatizan la comprensión más que la reproducción y fomentan la posibilidad de construir y reconstruir el conocimiento con los estudiantes desde lo aprendido, abriéndoles, así, espacios para una mayor participación (Durán 2001). 
Se podría inferir a partir de lo anterior que cualquier intento por mejorar los aprendizajes que ignore la importancia e impacto de estos aspectos implícitos en las prácticas evaluativas puede ver reducido sus efectos si los profesores no tienen conciencia respecto de su existencia e implicancias (Brown 2004). Ello, porque las creencias constituyen un soporte fundamental de sus prácticas profesionales y el trabajo docente requiere visibilizar los obstáculos que la frenan. Es preciso, en consecuencia, tomar conciencia respecto de la importancia de reflexionar críticamente sobre las creencias y dificultades implícitas en la realización del proceso evaluativo, dado que es "una actividad 'con mucho en juego' para alumnos, docentes y escuelas" (Earl y LeMahieu 2003: 213). Así lo establecen los nuevos planteamientos sobre la evaluación, al enfatizar la necesidad de transformar, entre otros cambios, las concepciones de los profesores sobre las capacidades de sus estudiantes (OECD 2005).

El valor de develar los referentes implícitos desde los cuales evalúan los profesores radica en el hecho que se constituyen como constructos para comprender e interpretar sus prácticas (Marcoleta, Flores y Seda 1997). Así se podría inferir que muchos de los problemas suscitados respecto de los resultados de los procesos formativos podrían provenir desde las creencias de los profesores que obstaculizan y/o posibilitan desarrollar una mejor docencia. Aceptando que estos referentes implícitos no pueden ser reducidos a simples enfoques interpretativos, tampoco es posible desconocer que para entenderlos es preciso identificarlos, dado que influyen tanto en la forma como significan los aspectos epistemológicos propios de su disciplina como en la manera de enseñarla. En efecto, los esquemas de significación construidos por los profesores les han configurado formas de interpretar y desarrollar sus prácticas docentes, lo que ha resultado en formas de trabajo de profundas y graves repercusiones que condicionan y califican las situaciones que viven y enfrentan cotidianamente los estudiantes en las aulas. Sobre esta base resulta particularmente importante analizar las concepciones y prácticas evaluativas de los profesores de Enseñanza Básica de Matemáticas y Lenguaje y Comunicación, pues su manera de enseñar y evaluar afectará y/o condicionará a los estudiantes por el resto de su itinerario escolar.

Concepciones y prácticas de evaluación en Matemáticas y Lenguaje y Comunicación en el nivel básico. El sentido de la enseñanza de los contenidos relacionados con los planes y programas de Educación Básica está referido a la construcción de conocimiento básico y al desarrollo de determinadas habilidades consideradas fundamentales para todos los estudiantes de ese nivel escolar, constituyendo, de esta forma, la base habilitante para otros aprendizajes a alcanzar en los diversos sectores y niveles superiores (Eisner 1994).

En el caso del subsector Lenguaje y Comunicación, estos programas establecen el desarrollo de habilidades relacionadas con la capacidad para comunicarse efectivamente (leer, escribir, escuchar y hablar); extraer información relevante; adoptar una postura crítica frente a lo que se lee; comprender los distintos medios de comunicación masiva; analizar y valorar obras literarias; seleccionar información desde distintas fuentes; producir textos funcionales; consolidar hábitos de lectura, etc. Las habilidades establecidas para el subsector de Matemáticas están relacionadas con la capacidad de resolver problemas cotidianos, a partir de la realización de operaciones básicas y procesos ordenados y sistemáticos; ubicarse espacialmente; identificar formas geométricas en su entorno cotidiano; desarrollar y emitir juicios matemáticos con fundamento; establecer regularidades y patrones en situaciones de la vida real, entre otros. 
Si se acepta que las prácticas evaluativas no son procesos meramente técnicos ni de control, es necesario cumplir con una serie de requisitos para que sus resultados reflejen efectivamente los aprendizajes de los estudiantes y el desarrollo de las respectivas habilidades. Ello implica que el profesor realice un complejo proceso de transformación del conocimiento disciplinario para su presentación didáctica, de manera de facilitar su comprensión por parte de los estudiantes; que defina los criterios de evaluación, diseñe instrumentos evaluativos en consonancia con lo anterior y determine cómo va a comunicar y trabajar sus resultados, articulando contenido disciplinario, su didáctica y la forma de evaluarlo. Las implicancias de este complejo proceso son fundamentales para el sentido de la evaluación, dado que las prácticas evaluativas están orientadas por redes semánticas y/o conceptuales referidas a la naturaleza y sentido de la disciplina, su enseñanza en general y de la evaluación en particular. Estas redes afectan y califican las situaciones y actividades de enseñanza y evaluación diseñadas para que los estudiantes construyan conocimiento, desarrollen sus habilidades y demuestren sus aprendizajes.

Concepciones y prácticas evaluativas de los profesores de Matemáticas. Muchos profesores de Matemáticas creen que esta disciplina es de naturaleza "objetiva", lo que les lleva a aplicar instrumentos evaluativos en los que los estudiantes deben realizar ejercicios de cálculos con operatoria básica, pruebas de memorización, selección múltiple o "problemas de planteo", todos los cuales requieren respuestas cortas y muy precisas (Alonso et al. 1996; Porlán y Rivero 1998, Romagnano 2000). Estos resultados son consistentes con los de un estudio nacional en el que se demostró que en el $2^{\circ}$ ciclo básico en el subsector de Matemáticas, las preguntas más frecuentes de los instrumentos evaluativos diseñados por los profesores estaban referidas a la aplicación y desarrollo de procedimientos, en los que los estudiantes debían resolver un problema o ejercicio matemático o científico siguiendo una fórmula o algoritmo sin necesidad de justificar el proceso (Unidad de Currículum y Evaluación del Ministerio de Educación. Documento de Trabajo 2006).

Sin embargo, los contenidos se deben evaluar contextualizándolos en la cotidianeidad vivida por los estudiantes, lo que amplía su sentido incluyendo aspectos más comprehensivos. A modo de ejemplo, se puede destacar que el contenido matemático incluye la ubicación espacial, lo que requiere privilegiar el desarrollo de estrategias didácticas y evaluativas que habiliten a los estudiantes a resolver este problema, entre otros similares y no sólo a demostrar el desarrollo de destrezas numéricas u operativas abstractas y descontextualizadas. Así lo establece el sentido de la evaluación "auténtica", que favorece la aplicación de conocimientos, destrezas y habilidades a situaciones que viven y experimentan cotidianamente los estudiantes (Aninat 2004; Himmel et al. 1999).

Un estudio acerca de las prácticas evaluativas en educación básica evidenció que los profesores de Matemáticas orientaban sus criterios de corrección por creencias asociadas al sentido tradicional de la evaluación, es decir, al control, dado que al momento de corregir y calificar sólo verificaban la existencia o no de la respuesta correcta predeterminada, sin solicitar la identificación del patrón o la descripción, caracterización, representación y resolución de problemas simples a partir de variables y operatorias explícitas (Saxe et al. 1997). Otro estudio mostró hallazgos similares, dado que los profesores creían en el sentido acreditador de la evaluación, por lo que solicitaban a los estudiantes realizar ejercicios de manera mecánica, privilegiando el cálculo y ordenación de secuencias 
(Delandshere y Jones 1999). Es decir, estos profesores no fomentaban ni desarrollaban el razonamiento, lo que les impidió tomar conciencia respecto de la necesidad de desarrollar este proceso con un sentido formativo.

Estas creencias y prácticas evaluativas también producen efectos incidentales, pues con más fuerza aún que la enseñanza, envían poderosos mensajes a los estudiantes acerca del contenido disciplinario que se evalúa, dado que por su intermedio los profesores relevan y enfatizan o privilegian ciertos conocimientos, habilidades y actitudes por sobre otras. Los resultados de un estudio así lo concluyen al develar que a los estudiantes de nivel básico les resultaba difícil comprender los criterios de evaluación, es decir, saber por qué un ejercicio está bien o mal resuelto, debido a que percibían que sus profesores/as sólo se fijaban en las "respuestas correctas o incorrectas", o "cuántos problemas he hecho bien y cuántos mal", "lo rápido que lo hago" y, en algunos casos, en aspectos formales tales como buena letra, orden y comportamiento (Bliem et al. 1995). Estas percepciones de los estudiantes pueden resultar en una representación distorsionada de la matemática al significarla como un conjunto de ejercicios desconectados entre sí, donde lo que se privilegia es el producto final más que el proceso y la cantidad y rapidez más que la calidad.

Los resultados de otro estudio revelan tensiones entre las concepciones de los profesores sobre la enseñanza y sobre la evaluación. En efecto, si bien ellos declaraban concepciones sobre la enseñanza más cercanas al constructivismo y reconocían, por ejemplo, que los estudiantes eran los actores centrales del proceso educativo, que las matemáticas debían tener una utilidad social y que los errores eran parte constitutiva del aprendizaje, no obstante, a la hora de evaluar controlaban el aprendizaje utilizando pruebas escritas, ya sea de selección múltiple o de respuesta breve, demostrando concepciones evaluativas tradicionales e instrumentales asociadas al control y verificación de los objetivos alcanzados (Gil 2004).

Se podría deducir que muchos profesores de matemáticas creen que su disciplina es de naturaleza objetiva y descontextualizada, lo que permea sus prácticas de enseñanza y les lleva a implementar procesos evaluativos reproductivos y mecánicos. Estas prácticas impiden a los estudiantes desarrollar su creatividad, sus habilidades argumentativas o aprender a fundamentar sus respuestas y, por lo tanto, a desarrollar sus habilidades para razonar, todos éstos, aspectos que representan algunos de los sentidos centrales del aprendizaje de las matemáticas.

Concepciones y prácticas evaluativas de los profesores de Lenguaje. En el área del Lenguaje, algunos profesores diseñan instrumentos evaluativos menos restrictivos y más abiertos, con distintos objetivos para distintos estudiantes, lo que les proporciona más posibilidades de reflexionar, emitir opiniones, realizar lecturas críticas y formular preguntas interesantes sobre lo enseñado (Black et al. 2003, Bliem y Davinroy 1997). Desde estos resultados se podría asumir, por lo tanto, que estos profesores orientarían sus prácticas por una concepción de evaluación formativa emancipadora que permite a los estudiantes construir conocimientos y participar más activamente en su proceso formativo.

Sin embargo, se ha detectado que la gran mayoría de los profesores evalúa la comprensión lectora a partir de segmentar el contenido en pequeños trozos organizados en una secuencia predeterminada, limitando la cobertura y evidencia de desempeño por parte de los estudiantes e impidiendo, en consecuencia, el desarrollo y comprobación de 
esta habilidad (Campbell 2001). Estos resultados estarían demostrando, por una parte, que muchos profesores de lenguaje no valoran la cohesión de las ideas, la coherencia con el texto, o la originalidad para expresar ideas (Solé et al. 2003). Por otra, sugiere que la única interpretación posible de lo leído es la de los profesores, sin permitir a los estudiantes evidenciar las propias, lo que les transmitiría la creencia que la comprensión lectora es dicotómica, es decir, se comprende o no un cierto texto o pasaje y, por lo tanto, ese texto adquiere estabilidad temporal de significados, ya que se presenta desvinculado de los contextos en los cuales se ha generado (Catalá et al. 2001; Campbell 2001).

A nivel del país la situación es bastante similar, pues se ha detectado que los profesores del área de Lenguaje y Comunicación desarrollan instrumentos evaluativos que privilegian el reconocimiento de características y acciones de personas, fenómenos o situaciones tal como aparecen en los textos leídos, minimizando de este modo la posibilidad de que los estudiantes emitan opiniones y establezcan relaciones. De hecho, las preguntas más frecuentes son las de alternativa simple, debiendo los estudiantes escoger una sola. En segundo lugar están las preguntas de desarrollo, que implican algún ejercicio de redacción, pero con un límite de líneas, con una extensión de 2 a 4,5 líneas, en su gran mayoría. Las preguntas de otro tipo, tales como completar o crear esquemas, construir mapas, realizar presentaciones o dibujos no alcanzan al $1 \%$. Es decir, lo que se privilegia en este subsector es la ejercitación de destrezas básicas, sin evidenciar preocupación por el desarrollo de las habilidades cognitivas superiores correspondientes a ese nivel y sector (Unidad de Currículum y Evaluación del Ministerio de Educación. Documento de Trabajo 2006). Desde estos resultados, se podría asumir que las prácticas evaluativas de los profesores nacionales estarían orientadas desde una racionalidad instrumental acerca de su naturaleza y sentido. Esta situación no es nueva en el país, dado que en un estudio realizado años atrás se comprobó que los profesores construían procedimientos de evaluación adscribiéndose a los libros de textos, enfatizando el refuerzo y privilegiando la memorización, entre otros aspectos, lo que impedía a los estudiantes demostrar su creatividad. Asimismo, planteaban la información descontextualizada de sus vidas cotidianas o desvinculada de sus conocimientos previos, lo que les impedía aplicarla a nuevas situaciones y, en definitiva, dificultaba su aprendizaje (Edwards 1994).

La situación antes descrita se ve agravada por un problema bastante frecuente en otras latitudes. Al igual que en el caso de los profesores de matemáticas, se han detectado inconsistencias entre las creencias que orientan la enseñanza y las que orientan los procesos evaluativos que desarrollan. En efecto, se ha constatado que aun cuando algunos profesores de enseñanza básica postulan expresamente la importancia del desarrollo de la comprensión lectora, establecen, no obstante, criterios evaluativos asociados a la verificación de la formalidad de la redacción y no a la calidad de dicha comprensión, por lo que administran instrumentos evaluativos que obstaculizan la comprobación del desarrollo de esta habilidad. De este modo, privilegian, en ocasiones, sólo la reproducción más o menos literal de los contenidos y, en otras, aprecian aspectos puramente expresivos o formales (Borko 1997).

Sin embargo, la comprensión lectora es un proceso dinámico, contextualizado social y culturalmente e interactivo. De esta manera, requiere ser evaluada no sólo con procedimientos que permitan comprobar la reproducción del contenido del texto sino que faciliten esta interacción entre el texto y el estudiante. Ello, porque la evaluación no puede limitarse sólo a la reproducción mecánica de un significado de un texto desvinculado de 
su contexto. Por el contrario, debería contemplar el desarrollo de habilidades relacionadas con su comprensión de textos acorde con el desarrollo de los estudiantes y la inferencia e interpretación de significados, incorporando textos de mayor funcionalidad tales como cartas, formularios, publicidades, entre otros (Atorresi 2005; Bono et al. 2000).

Dado lo anterior, existiría una contradicción entre las creencias de los profesores desde donde diseñan e implementan procesos de evaluación y la naturaleza de la comprensión lectora (Pérez 2005; Bono, Donolo y Rinaudo 2000). Si estas creencias resultan en determinadas prácticas, se deduce la necesidad de develarlas, no sólo por los importantes efectos en los aprendizajes de los estudiantes sino que particularmente para su desarrollo profesional y el consiguiente mejoramiento de su quehacer docente.

\section{CONCLUSIONES}

El interés de la sociedad por mejorar la calidad de la educación para que los estudiantes puedan construir los conocimientos y desarrollar las habilidades y actitudes significativas y relevantes para su vida presente y futura, ubica las prácticas de enseñanza y, de manera especial, a las evaluativas en un lugar central. Sin embargo, es necesario develar los múltiples factores que confluyen y que las condicionan, tales como los contenidos disciplinarios, los recursos didácticos, las relaciones interpersonales, el apoyo de los padres, las características socioculturales del entorno y, de manera crítica, las concepciones que sustentan los profesores, dada su gravitación en casi todas las actividades que se realizan en el aula.

Si bien es importante conocer los resultados del aprendizaje de los estudiantes es preciso entenderlos, por una parte, como una oportunidad para conocer sus dificultades y debilidades, ayudarles a superar los problemas detectados y habilitarlos para continuar sus estudios posteriores. Por otra, es preciso que los profesores los consideren como una fuente de relevante información para reorientar y transformar sus prácticas en pos del desarrollo de sus facultades. Lo anterior requiere destacar la importancia de conocer las racionalidades que están informando estas prácticas y develar las concepciones subyacentes, dado que permitiría identificar los conocimientos profesionales y/o creencias de los profesores y su valoración respecto de ciertos contenidos y ciertas habilidades.

Es fundamental, asimismo, desarrollar investigaciones sobre las concepciones de enseñanza y de evaluación que están informando las prácticas docentes de los profesores de Matemáticas y Lenguaje para develar las disonancias, contradicciones y repercusiones posteriores en sus estudiantes. Ello, porque estos profesores deben formar a todos los niños para desempeñarse lo mejor posible en su vida de adultos y los conocimientos que construyan y las habilidades que desarrollen en este nivel escolar deberán prepararlos para desenvolverse posteriormente, tal como lo indican los nuevos planteamientos sobre el sentido de la evaluación. Del mismo modo, facilitaría la transformación de las prácticas, facilitando el mayor nivel posible de desarrollo de las habilidades básicas de los estudiantes, facultándoles para elaborar construcciones cognitivas complejas, organizar y supervisar sus propios aprendizajes y proseguir sus estudios en los niveles siguientes.

Estos estudios podrían, asimismo, fortalecer el desarrollo de la evaluación como cuerpo de conocimientos, lo que facilitaría, a su vez, su contextualización en la realidad escolar. Asimismo, permitirían revisar los referentes que históricamente han informado las 
prácticas evaluativas, dado que podrían estar impulsando a muchos profesores a desarrollar determinadas prácticas, independientemente de la disciplina que enseñan, sin cuestionarse su sentido o funciones. También proporcionaría evidencias para analizar críticamente, tanto las políticas públicas que están enfatizando el desarrollo indiscriminado de pruebas estandarizadas nacionales e internacionales como las normativas, reglas y procedimientos provenientes de las mismas escuelas, dado que podrían estar influenciando las concepciones evaluativas de estos profesores $\mathrm{y}$, por ende, mediando sus prácticas.

\section{REFERENCIAS}

Alonso, A., D. Gil y J. Martínez-Torregrosa (1996). Concepciones docentes sobre la evaluación en la enseñanza de las ciencias. Alambique 4: 6-15.

Aninat, P. (2004). Matemática en el aula: Lo que nos falta por hacer. Revista de Educación 313: 23-29.

Atorresi, A. (2005). Competencias para la vida en las evaluaciones de Lectura y Escritura (SERCELLECE). Laboratorio latinoamericano de Evaluación de la Calidad de la Educación. http:// www.unesco.cl/medios/biblioteca/documentos/habilidades_para_vida_lenguaje_escritura.pdf

Avalos, B. (2003). La formación docente continua: discusiones y consensos. Diálogos Educacionales. Disponible en http://www.umce.cl/revistas/dialogoseducativos/dialogos_educativos_n4_ articulo_01.html

Bachelard, G. (1993). La filosofía del no, Buenos Aires: Editorial Amorrortu.

Black, P. et al. (2003). Assessment for Learning. Putting into Practice, London: Open University Press.

Bliem, C., K. Davinroy, V. Mayfield (1995). How does my teacher know what I know? Third Graders' perceptions of Math, Reading and Assessment, Technical Report 395 CRESST . Disponible en http://eric.ed.gov/ERICDocs/data/ericdocs2/content_storage_01/0000000b/80/27/3d/94.pdf

Bliem, C. y K. Davinroy (1997). Teachers' Beliefs About assessment and Instruction in Literacy. CSE Technical Report 421, CRESST. Disponible en http://www.cse.ucla.edu/products/Reports/ TECH421.pdf

Bolívar, A. (2000). La Mejora de los Procesos de Evaluación. Disponible en http://dewey.uab.es/ pmarques/dioe/DOEEva.rtf

Bolívar, A. y J. Segovia (2004). Competencias profesionales y crisis de identidad en el profesorado de secundaria en España. Perspectiva Educacional 44: 11-36.

Bono A., D. Donolo, M. C. Rinaudo (2000). La evaluación de la lectura. Cuestiones para pensar. Contextos de Educación IV. Disponible en http://www.unrc.edu.ar/publicar/cde/Bono,\%20 Donolo,\%20Rinaudo.htm

Borko, H., M. Flory y K. Cumbo (1993). Teacher's Ideas and Practices about Assesment and Instruction. A Case Study of the Effects in Instruction, Student Learning and Accountability Practices. CSE Technical Report 366 .CRESST. Disponible en http://www.cse.ucla.edu/products/Reports/TECH366.pdf

Borko, H. (1997). New Forms of Classroom Assessment: Implications for Staff Development. Theory into Practice 36: 231-238.

Brown, G. (2004). Teachers' conceptions of assessment: implications for policy and professional development. Assessment in Education 11, 3: 301-318.

Camilloni, A. (2005). La calidad de los programas de evaluación y de los instrumentos que los integran. En A. Camilloni et al. (eds.). La evaluación de los aprendizajes en el debate didáctico contemporáneo (pp. 9-30), Buenos Aires: Editorial Paidós Educador. 
Campbell, M. (2001). Inquiry into Reading Assessment: Teachers' perceptions of Effective Practices. Reading Horizons 42, 1: 1-20.

Carr, W. (1999). Una teoría para la educación. Hacia una Investigación Educativa Crítica. Madrid: Editorial Morata.

Carr, W., Kemmis, S. (1988). Teoría Crítica de la Enseñanza, Barcelona: Editorial Martínez de Roca.

Catalá, G. et al. (2001). Evaluación de la Comprensión Lectora, Barcelona: Editorial Graó.

Celman, S. (2005) ¿Es posible mejorar la evaluación y trasformarla en una herramienta de conocimiento? En A. Camilloni et al. (Comp.). La evaluación de los aprendizajes en el debate didáctico contemporáneo (pp. 35-66). Buenos Aires: Paidós Educador.

Chevallard, I. (1999). La transposición didáctica. Del saber sabio al saber enseñado, Madrid: Aique.

Darling-Hammond, L. (2001). El derecho de aprender. Crear buenas escuelas para todos, Barcelona: Ariel Educación.

Delandshere, G., J. Jones (1999). Elementary teachers' beliefs about assessment in mathematics: a case of assessment paralysis. Journal of Curriculum and Supervisión 14, 3: 216-240.

Díaz Barriga, A. (1993). El examen: texto para su historia y debate. México: UNAM.

Dutchasky, S. (1999). La escuela como frontera, Buenos Aires: Editorial Paidós.

Durán, E. (2001). Las creencias de los profesores: un campo para deliberar en los procesos de formación. Acción Educativa. Revista Electrónica. http://uas.uasnet.mx/cise/rev/Num1.

Edwards, V. (1995). El liceo por dentro: estudio etnográfico sobre prácticas de trabajo en Educación Media. Santiago: MINEDUC.

Earl, L. y P. LeMahieu (2003). Replantear la evaluación y rendición de cuentas. En A. Hargreaves (Comp.). Replantear el cambio educativo. Un enfoque renovador (pp. 211-236) Buenos Aires: Editorial Amorrortu.

Eisner, E. (1994). Cognición y Currículum. Buenos Aires: Editorial Amorrortu.

Eisner, E. (1998). El ojo ilustrado. Indagación y mejora de la práctica docente. Barcelona: Editorial Paidós.

Eraut, M. (1994). Developing Professional Knowledge and Competence, Londres: The Falmer Press.

Freire, P. (2002). Pedagogía de la autonomía. Buenos Aires: Editorial Siglo Veintiuno.

Frigeiro, G. (1998). La escuela en contextos turbulentos: aprendizajes y enseñanzas. Ensayos y Experiencias 22: 2-8.

Gil, F. (2000). Marco conceptual y creencias de los profesores sobre evaluación en matemáticas, Almería: Ediciones Universidad de Almería.

Gómez Chacón, I. (2000). Matemática emocional. Los afectos en el aprendizaje matemático. España: Ediciones Narcea.

Himmel, E., M. A. Olivares y J. Zabalza (1999). Hacia una Evaluación Educativa. Aprender para Evaluar y Evaluar para Aprender. Vol. I. Santiago: PUC y MINEDUC.

Jackson, Ph. (2002). Práctica de la enseñanza. Buenos Aires: Editorial Amorrortu.

Kaplan, C. (2004). La inteligencia escolarizada. Buenos Aires: Editorial Miño y Dávila.

Liston, D. y K. Zeichner (1993). Formación del profesorado y condiciones sociales de la escolarización. Madrid: Editorial Morata.

Litwin, E. (2005). La evaluación: campo de controversias y paradojas o un nuevo lugar para la buena enseñanza. En A. Camilloni et al. (2005). La evaluación de los aprendizajes en el debate didáctico contemporáneo (pp. 11-34), Buenos Aires: Editorial Paidós Educador.

Marcelo, C. (2002). Aprender a enseñar para la sociedad del conocimiento. Education Policy Análisis 10, 35. Disponible en http://epaa.asu.edu/epaa/v10n35/ 
Marcoleta, S., R. Flores e I. Seda (1997). Las creencias de docentes mexicanos sobre el papel de la escuela y del maestro. Revista Iberoamericana de la Educación. OEI. Disponible en http:www.campus-oei.org/deloslectores/marcoleta. pdf

Martínez, F. (2004). El sentido de la evaluación en Educación Básica. Revista Mexicana de Investigación Educativa 9, 23: 817-839.

Martínez Padros, O. (2005). Dominio afectivo en educación matemática. Paradigma 26, 2: 7-34.

Mellado, V. (1996). Concepciones y prácticas de aula de profesores de ciencias, en formación inicial de Primaria y Secundaria. Enseñanza de las Ciencias 14, 3: 289-302.

Mineduc (2006). Evaluación de aula en enseñanza Básica y Media. Documento de Trabajo Equipo de Seguimiento a la Implementación Curricular. Unidad de Currículum y Evaluación.

Montero, L. (2001). La construcción de conocimiento profesional docente, Argentina: Editorial Homo Sapiens.

Moreno, M. y C. Azcárate (2003). Concepciones y creencias de los profesores universitarios de matemáticas acerca de la enseñanza de las ecuaciones diferenciales. Enseñanza de las Ciencias 21, 2: 265-280.

Nespor J. (1987). The role of beliefs in the practice of teaching. Journal of Curriculum Studies 19, 4: 317-328.

OECD (2005). Formative Assessment. Improving Learning in Secondary Classroom. París: OECD.

Pajares, M. (1992). Teacher's beliefs and educational research: cleaning up a messy construct. Review of Educational Research 6, 3: 307-332.

Palau de Mate, M. (2005). La evaluación de las prácticas docentes y la autoevaluación. En A. Camilloni (comp.). Los obstáculos epistemológicos de la enseñanza (pp. 93-132), Buenos Aires: Editorial Geodisa.

Pérez, M. J. (2005). Evaluación de la Comprensión Lectora: Dificultades y limitaciones. Revista de Educación, $\mathrm{N}^{\circ}$ extraordinario: 121-138.

Peterfalvi, B. (1997). Identificación de los obstáculos por parte de los alumnos. En A. Camilloni (comp.). Los obstáculos epistemológicos en la enseñanza (127-168), Barcelona: Editorial Geodisa.

Ponte, J. P. (1992). Concepções dos professores de matemática e processos de formação. In J. P. Ponte (ed.), Educação matemática: Temas de investigação (pp. 185-239). Lisboa: Instituto de Inovação Educacional. Disponible en http://www.educ.fc.ul.pt/docentes/jponte

Porlán, R. y A. Rivero (1998). El conocimiento de los profesores. El caso de la enseñanza de las ciencias. Sevilla: Editorial Díada.

Prieto, M. (2005). La participación de los estudiantes: ¿un camino hacia su emancipación? Theoria 14, 1: 27-36.

Romagnano, L. (2001). The myth of objectivity in mathematics assessment. The Mathematics Teacher 94, 1: 321-313.

Santos Guerra, M. A. (1996). Evaluar es comprender: De la concepción técnica a la dimensión crítica. Investigación en la Escuela 30: 5-13.

Saxe, G. et al. (1997). Teachers' Shifting Assesment Practices in the Context of Educational Reform in Mathematics. CSE Technical Report 471, CRESST. Disponible en http://www.cse. ucla.edu/products/Reports/TECH471.pdf

Shor, I. (1992). Empowering Education. Chicago: The Chicago Press.

Shön, D. (1992). La formación de profesionales reflexivos: Hacia un nuevo diseño de la enseñanza y el aprendizaje en las profesiones. Madrid: Paidós/Mec.

Shulman, L. (1987). Knowledge and teaching: Foundations of the new reform. Harvard Educational Review 57, 1: 1-22. 
Solé, I., M. Miras y N. Castells (2003) ¿Dónde se encuentra la innovación en las prácticas de evaluación innovadoras? Infancia y Aprendizaje 26 (2): 217-233.

Stenhouse, L. (1998). La investigación como base de la enseñanza. Madrid: Editorial Morata.

Stiggins, R. (2004). New Assessment Beliefs for a New School Mission, Phi Delta Kappan 86, 1: 22-27.Disponible en http://www.pdkintl.org/kappan/k_v86/ktoc0409.htm

Stiggins, R. (2006). What a difference a word makes. Assessment FOR learning rather than assessment OF learning helps students succeed. Journal of Staff Development 27, 1: 10-14. Disponible en http://www.nsdc.org/library/publications/jsd/stiggins271.pdf

Torrance, H. y Pryor, J. (1998). Investigating formative assesment. Teaching, learning and assesment in the classroom Buckingham: Open University Press.

Torrance H. y J. Pryor (2001). Developing Formative Assessment in the Classroom: Using Action Research to Explore and Modify Theory. British Educational Research Journal 25, 5: 615631.

Wang, P. (2004). Chinese science teachers beliefs and practices of assessment. Ph.D Disssertation. University of Georgia, Athens. Disponible en http://purl.galileo.usg.edu/uga\%5Fetd/ wang\%5Fping $\% 5 \mathrm{~F} 200412 \% 5 \mathrm{Fphd}$ 

Las colaboraciones, pedidos, suscripciones y correspondencia deben ser dirigidas a la Dirección de la revista: Oficina de Publicaciones, Facultad de Filosofía y Humanidades, Universidad Austral de Chile, Casilla 567, Fono/ Fax: 5663 221275, Valdivia, Chile. E-mail: eped@uach.cl

Las suscripciones incluyen los gastos de envío:

Chile: \$2.000. Otros países: US\$ 10.

El canje debe ser enviado a Biblioteca Central, Universidad Austral de Chile,

Correo 2, Valdivia 